\title{
Zum Formwandel der Internationalisierung bei VW in den 80er und 90er Jahren
}

\section{Einleitung}

In der Standortdebatte der 90er Jahre bündeln sich die unterschiedlichen Perspektiven und Interessenpositionen in der Frage, welche Konsequenzen sich aus den Prozessen der Globalisierung und Internationalisierung für die nationalen Institutionensysteme ergeben (Simons, Westermann 1997). In diesem Zusammenhang spielt neben der Globalisierung der Finanzmärkte die Internationalisierung der Produktion eine entscheidende Rolle. ${ }^{1}$ Es sind vor allem die Direktinvestitionsströme, anhand derer die Qualität eines Standorts bewertet wird. In der Argumentation gilt die einfache Gleichung: je weniger Direktinvestitionen aus dem Inland abgeflossen und je mehr dem Inland zugeflossen sind, desto besser ist die Position des Standorts in der internationalen Arbeitsteilung. Abhängig von der Einschätzung des Betrachters werden aus dem errechneten oder prognostizierten Saldo politische Schlußfolgerungen hinsichtlich der Verbesserung der Standortqualität gezogen, die sich entweder auf die Lohn- und Steuerhöhe oder auf weiterreichende institutionelle Veränderungen beziehen.

In historischer Perspektive überrascht sowohl die Schärfe als auch die Stoßrichtung der Diskussion. Denn eine Internationalisierung der Produktion hat es während der gesamten Nachkriegsentwicklung gegeben, ohne daß sich daran eine umfassendere politische und wissenschaftliche Grundsatz-

1 Es dürfte als ein Minimalkonsens der Globalisierungs- und Standortdebatte gelten, daß sinnvollerweise zwischen der Globalisierung der Finanzmärkte und der Internationalisierung der Produktion zu unterscheiden ist. Während die monetäre Globalisierung sich auf die Entstehung deregulierter internationaler Finanzmärkte bezieht, auf denen Aktien, Wertpapiere, Währungen und Derivate gehandelt werden, steht bei der Internationalisierung die Entwicklung ausländischer Direktinvestitionen von Unternehmen des Industrieund Dienstleistungssektors im Zentrum des Interesses. Es wird von der Internationalisierung der Produktion und nicht von ihrer Globalisierung gesprochen, weil sich die Produktion bislang noch nicht in dem Maße weltumspannend globalisiert hat, wie dies für die internationalen Finanzmärkte gilt. Zwar sind die weltweiten ausländischen Direktinvestitionen seit Mitte der 70er Jahre erheblich angestiegen, konzentrieren sich aber im Aggregat noch vorwiegend auf die entwickelten Triadenökonomien (vgl. Hickel 1996). 
debatte über die Qualitäten und die institutionelle Verfassung des Standorts ergeben hätte. Die Internationalisierung war bis in die 70er Jahre ein Thema, das in erster Linie von Marxisten verfolgt wurde, die sich für den $\mathrm{Zu}$ sammenhang von Internationalisierung und ökonomischer Macht oder für weltwirtschaftliche Ungleichheiten interessierten. Seit den 80er Jahren wird die Internationalisierung der Produktion jedoch vor allem von konservativen Interessengruppen als Argument für institutionelle Deregulierungen stark gemacht. Wie läßt sich diese auffällige Verschiebung der Rezeption und der Bewertung von Internationalisierungsprozessen erklären? Steckt dahinter bloß ein willkürlicher Interpretationsakt der Betrachter, oder liegt der Verschiebung eine reale Veränderung des Gegenstandes zugrunde?

In diesem Aufsatz sollen anhand der Entwicklung des Volkswagen-Konzerns einige Befunde zur neuen Qualität der Internationalisierung vorgestellt werden. Der Volkswagen-Konzern ist der größte europäische Automobilhersteller und auch nach der Fusion von Chrysler und Daimler-Benz immer noch der viertgrößte Produzent der Welt (gemessen am Produktionsvolumen). Die Unternehmensstatistiken weisen seit der zweiten Hälfte der 80er Jahre einen signifikanten Internationalisierungstrend aus, der sich gleichermaßen in Absatz, Produktion und Beschäftigung niederschlägt (Geschäftsberichte VW AG, div. Jahrgänge). So stieg im Zeitraum von 1985 bis 1997 das Verhältnis von Ausland und Inland in der Größe Absatz von $232 \%$ auf $328 \%$ an, in der Größe Produktion von $47 \%$ auf $165 \%$ und in der Größe Beschäftigung von 52\% auf 91\%. Stellt man bei der Beschäftigung die 1994 eingeführten rigorosen Arbeitszeitverkürzungen an den Inlandsstandorten der VW AG in Rechnung, so dürfte die Beschäftigung an den Auslandsstandorten die Inlandsbeschäftigung gemessen in Stundenarbeitsvolumen inzwischen überholt haben. Ein weniger einheitliches Bild ergeben auf den ersten Blick die Investitionen, die stärker schwanken und bei denen sich das Verhältnis von Auslands- zu Inlandsinvestitionen zwischen 1985 und 1997 lediglich von 35\% auf 40\% erhöht hat. Dieses Bild relativiert sich bei näherer Betrachtung jedoch erheblich. Zum einen ist das Jahr 1997 nur sehr bedingt als Referenzjahr aussagekräftig, weil in den Vorjahren der Anteil der Auslandsinvestitionen durchweg höher war. Zum anderen sind ein Großteil der Inlandsinvestitionen als Finanzinvestitionen der Konzernmutter, der deutschen VW AG, verdeckte Auslandsinvestitionen. So betrug beispielweise der Anteil der Sachinvestitionen an den Inlandsinvestitionen im Jahr 1994 gerade 12\%, während 88\% der Inlandsinvestitionen Finanzinvestitionen waren, die zu einem großen Teil an die ausländischen Konzerntöchter gingen. Der quantitative Internationalisierungstrend läßt sich daher auch für die Investitionen feststellen.

Doch die Entwicklung der Zahlen ist nur eine Seite des Prozesses. Die andere Seite sind die qualitativen Veränderungen der Internationalisierung, 
die sich hinter den Zahlen verbergen. In der folgenden Analyse werden zwei Kernpunkte der Internationalisierung herausgearbeitet. Erstens wird gezeigt, daß die Internationalisierung der Produktion mehr ist als die nur quantitative Ausdehnung der ausländischen Direktinvestitionen transnationaler Unternehmen. Mit der Internationalisierung der 80er und 90er Jahre ist ein Formwandel der Produktion verbunden, der nicht nur die Organisationsstrukturen der transnationalen Unternehmen nachhaltig verändert, sondern die gesamte Organisation der Produktionskette umfaßt. Dieser Formwandel kann als Übergang vom multinationalen Konzern zum »global atmenden Produktionsnetzwerk ${ }^{2}$ beschrieben werden. Daran anknüpfend kann zweitens festgestellt werden, daß die Internationalisierung eine moderne Variante der Konzentration ist. Konzentration verläuft immer mehr in Formen internationaler Akquisitionen, der internationalen Ausdehnung von Kernkompetenzen und internationaler Vernetzungen, aber immer weniger in Formen der vertikalen Integration oder der Diversifizierung. Die Internationalisierung als Konzentration ist eine aktive Konkurrenzstrategie, die zur Intensivierung der Konkurrenz und nicht zu ihrer Überwindung beiträgt.

\section{Eine Formanalyse der Internationalisierung und der Konzentration}

Vor dem Einstieg in die qualitative Analyse der Internationalisierungsprozesse ist kurz der zugrunde liegende Formbegriff zu erläutern. Der Formbegriff knüpft an neuere marxistische und institutionalistische Ansätze an, die an der Analyse historischer Institutionen orientiert sind. An erster Stelle ist dabei die Regulationstheorie zu nennen, die sich um die Konzepte »Regulation «, »Akkumulationsregime « und »Entwicklungsmodell « zentriert (vgl. dazu Hübner 1989, Boyer 1990, Hollingsworth u.a. 1994):

- Institutionelle Formen sind komplexe Ensembles aus Institutionen, die über unterschiedliche Regulierungsmechanismen verknüpft sind.

- Eine institutionelle Form ist in der Zeit wandelbar, ihre Stabilität ist transitorisch und ihr Entstehen eher unwahrscheinlich.

- In der Formanalyse geht es um fragile und historische Kohärenzen oder Entsprechungsverhältnisse zwischen Institutionen.

Für die Formanalyse von Unternehmensprozessen liefert die Regulationstheorie in ihren früheren Ansätzen zwar mit dem Begriff des »technologischen Paradigmas « erste Anknüpfungspunkte, doch bezieht sich der Begriff zu eng auf die technologische und arbeitsorganisatorische Dimension der Produktion. Weder werden damit die Formen der Unternehmens- oder Betriebsorganisation noch die Formen der Internationalisierung oder der

2 So eine Selbstbeschreibung von VW, die einen durchaus wesentlichen Sachverhalt trifft. 
zwischenbetrieblichen Beziehungen erfaßt. Diese Leerstelle kann mit dem von Robert Boyer entwickelten Begriff des »Produktionssystems « geschlossen werden (Boyer, Durant 1997). Ein Produktionssystem ist ein Ensemble aus den angewandten markt-, produkt- und produktionsstrategischen Managementprinzipien, der Unternehmensorganisation und den industriellen Beziehungen im Unternehmen. Die Internationalisierung ist in ihrer strategischen und organisatorischen Dimension ein integriertes Element eines Produktionssystems. Zugleich fließen sowohl die zwischenbetrieblichen Beziehungen als auch die Beziehungen zu den gesellschaftlichen Meso- und Makroinstitutionen in das Produktionssystem ein.

Das Produktionssystem weist über die institutionelle Analyse hinaus auf die durch die Institutionen regulierten sozialen Beziehungen. Strategien und Prinzipien werden von Akteuren verfolgt, Organisationsformen sind nur die formelle Seite einer sozialen Organisation von Akteuren. David Lockwod hat mit seiner Unterscheidung von System- und Sozialintegration auf den hohen Stellenwert der sozialen Interaktionen in der sozialwissenschaftlichen Analyse aufmerksam gemacht (Lockwood 1964). Erst auf der Ebene der Sozialintegration, der sozialen Interaktionen zwischen den Akteuren, entscheidet sich, ob die Akteure bestehende Institutionen reproduzieren oder ob sie diese in einem bewußten Akt oder als unintendierte Nebenfolge ihres Handelns verändern. In der durch die Interessen und Normen der Akteure geprägten sozialen Interaktion entwickeln die Akteure ihre Deutungen und Interpretationen der Wirklichkeit und ihre situativen Lösungsansätze für wahrgenommene Probleme. System- und Sozialintegration verbindet eine Akteurskausalität, die dadurch ensteht, daß Institutionen soziale Konstruktionen sind und daß soziales Handeln nicht durch die Systembedingungen determiniert wird, sondern Freiheitsgrade besitzt, die mit dem Begriff der Kreativität belegt werden können (Joas 1992).

Schließlich muß die Analyse von Produktionssystemen die Besonderheiten nationaler Institutionensysteme berücksichtigen, die jenseits allgemeiner Systembedingungen besondere nationale Entwicklungspfade nach sich ziehen (Berger, Dore 1996). Gerade das bundesdeutsche Produktionsmodell der Nachkriegszeit wies vielfältige Differenzen gegenüber dem in den Industrieländern verbreiteten fordistischen Modell auf (vgl. zum Begriff des Fordismus Lipietz 1985). Diese Differenzen erstreckten sich über die duale Struktur der bundesdeutschen Ökonomie (Herrigel 1996), institutionelle Besonderheiten des Finanz- und des Innovationssystems (Soskice 1994) und das auf den tragenden Säulen der Tarifautonomie und der Mitbestimmung ruhende duale System der industriellen Beziehungen (Thelen 1992). Vor allem das duale System der industriellen Beziehungen mit den von den Industrie- und Branchengewerkschaften sowie den Arbeitgeberverbänden zentralisierten Tarifverhandlungen und den rechtlich verankerten Mitbe- 
stimmungsorganen und Mitbestimmungsrechten auf Unternehmens- und Betriebsebene prägt unmittelbar die industriellen Beziehungen und die Arbeitsbeziehungen im Betrieb und weist deutliche Differenzen zu allen anderen nationalen Systemen industrieller Beziehungen auf (Rogers, Streeck 1995, Thelen, Turner 1997).

\section{Multinationale Internationalisierung bis Ende der 70er Jahre}

Volkswagen hatte in den 50er und 60er Jahren ein fordistisches Produktionssystem par excellence entwickelt, dessen konstitutive Elemente kurz zusammengefaßt die fordistische Massenproduktion, die tayloristische Arbeitsorganisation, eine hierachisch-funktional-zentralistische Betriebs- und Unternehmensorganisation, eine hohe vertikale Integration, multinationale Konzernstrukturen und industrielle Beziehungen waren, die sich durch eine breite Akzeptanz fordistischer Rationalisierung und konfliktuelle Beziehungen hinsichtlich der materiellen Verteilung der Produktivitätsgewinne auszeichneten (Boyer 1997, 7ff.; für das folgende Wellhöner 1996). Zu Beginn der 50er Jahre entschied sich die damalige Unternehmensleitung unter dem Generaldirektor Heinrich Nordhoff zu einer exportorientierten Ein-Produkt-Strategie. Die unbestrittenen Produktivitätsvorteile der amerikanischen Automobilproduzenten in den 50er Jahren veranlaßten das Management zur Übernahme der modernen amerikanischen Massenproduktionstechnologien, begünstigt durch die Konzentration auf die Produktion des Käfers. In den Jahren ab 1954 hielt die fordistische Fließfertigung mit ihren Elementen Fließband, Einzweckmaschinen und Transferstraßen nach und nach Einzug in das Wolfsburger Volkswagenwerk. Zugleich wurden die tayloristischen Formen der Arbeitsorganisation eingeführt. Rigide Arbeitsteilung, Methoden- und Zeitstudien und die Trennung von Ausführung und Disposition bestimmten von da an die Organisation der Arbeit. Bald zeigte sich, daß die alte Organisationsstruktur des Unternehmens mit den neuen Produktionskonzepten nicht mehr kompatibel war, schon weil durch den Taylorismus viele indirekte Funktionen von der Produktion entkoppelt wurden, die neu organisiert werden mußten. In dieser Situation entschied sich das Management für die Implementierung einer funktional-zentralistischen Organisationsform nach dem Vorbild amerikanischer Großkonzerne. Die großen Markterfolge der Käfer-Produktion und die Strategie der Massenproduktion sprengten rasch den Rahmen des Werkes Wolfsburg, weil erstens die Massenproduktionstechnologie sehr raumintensiv war und zweitens die Unternehmensleitung frühzeitig auf eine Strategie vertikaler Integration setzte. Weil zudem eine Nutzfahrzeugproduktion aufgenommen wurde, mußte mit dem Transporterwerk in Hannover bereits 1954 ein neues Werk gegründet werden, in dem zunächst auch die Produktion von 
Hinterachsen und Motoren aufgenommen wurde. Rasch folgten der Ausbau des ehmaligen Ausbildungswerkes Braunschweig, die Gründungen der Werke Kassel, Emden, Salzgitter und der europäischen Tochtergesellschaft in Brüssel. Von diesen Werken waren Emden und Brüssel Endmontagewerke, die anderen Werke waren als interne Komponentenfertiger Ausdruck einer hohen Fertigungstiefe, mit der die positiven Skaleneffekte einer massenproduzierenden Komponentenfertigung für Volkswagen erschlossen werden sollten. Die vertikale Integration und die Ausdehnung der Produktion waren die zentralen Formen der Konzentration dieser Entwicklungsphase. Sie wurden ergänzt durch die Akquisition der Auto-Union AG, die im Jahre 1966 mit den NSU Motorenwerken zur Audi NSU Auto Union AG verschmolzen und dem Unternehmen als Tochtergesellschaft eingegliedert wurde. Das Unternehmen wollte durch den Erwerb die selbst nur mit großer Mühe und mit wenig Erfolg betriebene Differenzierung der Modellpalette vorantreiben, weil ein Ende des Käfer-Booms langsam absehbar wurde. Neue Modelle sollten sowohl den Käfer unterstützen als auch neue Marktsegmente in einem sich differenzierenden Automobilmarkt erschließen. Die Akquisition diente daher nicht der Einnahme einer marktbeherrschenden Stellung, sondern war eine Reaktion auf die sich langsam verändernden Marktbedingungen.

Die Internationalisierung des Volkswagenwerkes in den 50er und 60er Jahren hatte mit den elementaren Konzepten des fordistischen Produktionsmodells wenig zu tun. Sie diente allein der Erschließung politisch regulierter Märkte, in denen Marktpräsenz nur durch Auslandsproduktion erreicht werden konnte. Volkswagen errichtete in den 50er und 60er Jahren Produktionsstätten in Brasilien, Australien, Südafrika und Mexiko. Diese Produktionsstandorte wurden als Tochtergesellschaften organisiert, die relativ autonom von der Muttergesellschaft, der Volkswagenwerk AG in Wolfsburg, in ihren regionalen Märkten operierten. Volkswagen nahm das Profil eines multinationalen Konzerns mit ausgeprägter Mutter-TocherTrennung und regionalen Strukturen an.

Die industriellen Beziehungen des Unternehmens entwickelten einen kooperativen Charakter, seit sich die IG Metall im Verlauf der 50er Jahre als dominierende Kraft in den Betriebsverfassungsorganen durchgesetzt hatte und der Betriebsrat aufgrund seiner engen gewerkschaftspolitischen Anbindung und der festen Verankerung in einer immer mehr gewerkschaftlich organisierten Belegschaft erstarkte (Koch 1987). Zwischen Interessenvertretung und Unternehmensleitung entstand ein Rationalisierungspakt, der auf einer »zivilisierten « (Kern 1997), an der Verbesserung der Produktionsmethoden und nicht an der Arbeitsintensität ansetzenden Rationalisierung und auf hohen Löhnen und Sozialstandards beruhte. Während die Interessenvertretung die fordistische Rationalisierung akzeptierte, gewährte 
die Unternehmensleitung materielle Konzessionen. Volkswagen wurde ein Vorreiter bei der tarifvertraglichen Ausgestaltung von Lohn- und Arbeitszeitpolitik. Der kooperative Charakter der industriellen Beziehungen wurde lediglich in der Krise Mitte der 70er Jahre im Angesicht drohender Massenentlassungen kurzfristig unterbrochen, prägt seitdem aber weiterhin das Bild der Beziehungen.

Die Krise Mitte der 70er Jahre traf das Unternehmen deshalb mit besonderer Wucht, weil sich vier Strukturprobleme überlagerten. Das erste Strukturproblem war die allgemeine Krise der fordistischen Akkumulation, in deren Zentrum die Krise des fordistischen Produktionssystems stand. Das fordistische Produktionssystem war an seine sozialen, technologischen und ökonomischen Grenzen gelangt, weil die Produktivitätsspielräume des Modells ausgereizt waren und sich seine technologischen und organisatorischen Strukturen zunehmend mit den neuen Erfordernissen einer intensivierten Konkurrenz auf differenzierten und integrierten Märkten brachen (Boyer 1997, 11ff.). Flexibilität, Qualität, Kosten und Zeit lauteten die neuen Wettbewerbsparameter. Das fordistische Produktionssystem entsprach diesen neuen Paramtern vor allem deshalb nicht mehr, weil die für standardisierte Massenproduktion ausgelegten Technologien und Organisationsstrukturen die schnelle und flexible Produktion differenzierter und qualitativ hochwertiger Produkte nicht effizient umsetzen konnten. Es entstand ein Rationalisierungsdilemma zwischen Effizienz und Flexibilität. Das zweite Problem war die Käfer-Monostruktur des Unternehmens, die den veränderten Marktbedingungen nicht mehr gerecht wurde. Der Erwerb der Audi NSU Auto-Union AG und die Einführung einer neuen Produktpalette mit den Modellen Passat, Golf, Scirocco und Polo kamen zu spät, um die Situation entscheidend verbessern zu können. Drittens war der Konzern seit seinen großen Exporterfolgen im US-Markt in den 50er und 60er Jahren hochgradig vom US-Export abhängig und deshalb von der kontinuierlichen Aufwertung der D-Mark nach dem Zusammenbruch des Weltwährungssystems von Bretton-Woods besonders betroffen. Schließlich wirkte die Ölkrise und die Erhöhung der Benzinpreise gerade für die Automobilindustrie zusätzlich nachfragesenkend.

Die Unternehmensleitung reagierte auf die Krise mit einer zweigleisigen Strategie. Neben der zügigen Einführung der neuen und differenzierten Produktpalette, die aus der Forschung und Entwicklung der Audi NSU Auto-Union stammte, sollte die Errichtung eines Produktionswerkes in den USA die Abhängigkeit des Unternehmens von Währungsschwankungen reduzieren und die Position des Unternehmens auf dem US-Markt stabilisieren. Auf dem Höhepunkt der Krise in den Jahren 1974 und 1975 mußte dieses Projekt jedoch verschoben werden. Statt dessen drohten Massenentlassungen, die vom 1975 gewählten neuen Vorstand unter Toni Schmücker 
gegen die Stimmen der Arbeitnehmervertreter im Aufsichtsrat durchgesetzt wurden. Die Massenentlassungen konnten durch Aufhebungsverträge und die rasche Besserung der konjunkturellen Situation ab 1976 abgewendet werden, und die US-Strategie kam erneut auf die Tagesordnung. In der zweiten Hälfte der 70er Jahre hatten sich die Vorzeichen für das Projekt allerdings gewandelt. Zwar blieben die Abschottungen gegen Wechselkursturbulenzen und die Sicherung des US-Marktes zentrale Motive für das Projekt, doch trat erstmals das Kostenmotiv in den Vordergrund. Volkswagen war auf dem US-Markt in den Sog der japanischen Konkurrenz geraten, gegenüber deren Preisoffensiven das Unternehmen von seinen europäischen Produktionsstandorten aus nicht mehr konkurrenzfähig war (Streeck 1984, 57). Erstmals knüpften Interessenvertretung und Arbeitnehmervertreter im Aufsichtsrat angesichts der Krisenerfahrungen der 70er Jahre ihre Zusage für das Projekt an Beschäftigungsgarantien für die inländische Beschäftigung, weil sie erkannt hatten, daß sich mit dem US-Projekt eine neue Form der Internationalisierung andeutete, deren Logik die Ausnutzung standortbedingter Kostenvorteile war.

Das Unternehmen entwickelte in der zweiten Hälfte der 70er Jahre nicht nur neue Strategien der Internationalisierung, sondern auch eine neue Strategie der Konzentration. Mit der Übernahme des Schreibmaschinenherstellers Triumph-Adler startete Volkswagen den Versuch einer Diversifikation der Konzernstrukturen in einen anderen Produktbereich. Ausgangspunkt dafür war die in der Struktur- und Energiekrise der 70er Jahre gewonnene Skepsis der Unternehmensleitung hinsichtlich der langfristigen Zukunftsfähigkeit massenhafter Automobilproduktion. Die Eröffnung einer neuen Produktsparte sollte dem Konzern ein zweites und zukunftsträchtiges Standbein verschaffen. Doch der Versuch diversifizierter Konzernbildung war die Geschichte eines kostenträchtigen Scheiterns. Weder spielten sich zwischen den Konzernsparten Synergieffekte ein, noch konnte die neue Tochter bei der mikroelektronischen Revolutionierung der Datenverarbeitung technologisch Anschluß halten. Mit dem Verkauf der Tochter an Olivetti im Jahr 1986 endete der Versuch der diversifizierten Konzernbildung und wurde seitdem nicht mehr aufgenommen.

\section{Konzentration als Internationalisierung und die Unternehmensstrategie der 80er Jahre}

Im Zeitraum Ende der 70er und Anfang der 80er Jahre schälten sich unter dem Vorstandsvorsitzenden Toni Schmücker und ab 1982 unter seinem Nachfolger Carl H. Hahn die Konturen einer neuen Unternehmensstrategie heraus, mit der die Unternehmensleitung den gewandelten Herausforderungen der Märkte begegnen wollte. Dabei war das zentrale Problem die Ver- 
knüpfung von Flexibilität und Effizienz. Um dieses Rationalisierungsdilemma der Massenproduktion kreisten die vier Elemente der Unternehmensstrategie.

Das erste Element war die Strategie technologischer Rationalisierung. Sie setzte auf die Flexibilitätsspielräume neuer Technologien. Im Zentrum standen die Robotertechnologie und mikroelektronische Informations- und Steuerungstechnologien. Konstruktion, Produktion und Logistik sollten durch den Einsatz flexiblerer Technologien an die neuen Flexibilitätserfordernisse hinsichtlich Mengen, Typen und Varianten angepaßt werden. Das Flexibilitätsdilemma der fordistischen Technologie sollte technologisch aufgelöst werden. Die Roboterisierung erreichte mit der Einweihung der berühmten, zu ca. 25\% automatisierten Montagehalle 54 im Jahre 1984 einen Höhepunkt, nachdem sich die Robotertechnologie zuvor auf Rohbau, Lackiererei und Preßwerk konzentriert hatte. CAM- und CAD-Systeme und zentrale Logistiksteuerungssysteme wurden als mikroelektronische Informations- und Steuerungstechnologien eingesetzt, um die Konstruktion zu beschleunigen, die Abstimmung zwischen Konstruktion und Produktion zu verbessern und den Teilefluß zu systematisieren. Das langfristige Ziel war die Integration der unterschiedlichen Informationssysteme in eine umfassende und zentrale CIM-Steuerung aller Aktivitäten des Unternehmens.

Die Volumenstrategie als zweites Element hatte zwei Stoßrichtungen. Zum einen sollte über die Ausnutzung aller sich bietenden Marktchancen die Produktion so weit erhöht werden, daß die Skaleneffekte der Massenproduktion trotz steigender Varianten- und Typenvielfalt weiterhin realisiert werden konnten. Zum anderen wurde aggressiv die Marktführerschaft in Westeuropa angestrebt, weil der Imagegewinn des Marktführers höhere Preispositionierungen der Produkte erlaubte. Diese auf etwa 5\% geschätzten Preisspielräume waren für das Unternehmen deshalb so wichtig, weil zu Beginn der 80er Jahre Kostenprobleme aufgetreten waren, die das Unternehmensergebnis erheblich beeinträchtigten.

Das dritte Strategieelement, die Kostensenkungsstrategie, zielte auf die Beseitigung dieser Kostenprobleme. Bereits in der Krise der Jahre 1981-1983 wurde ein Fixkostendruck spürbar, der den Ergebnisbeitrag aufzuzehren drohte. Weil aufgrund der Strategie der technologischen Rationalisierung die investitionsabhängigen Kosten unter den Fixkosten kaum beeinflußbar waren, richteten sich die Kostensenkungsbestrebungen der Unternehmensleitung auf die Personalgemeinkosten, die in der fordistischen Organisation durch die Aufblähung der indirekten Bereiche stark angewachsen waren. Die hohen Gemeinkosten sollten durch Personalabbau gesenkt werden, zunächst mit den Mitteln einer differenzierteren Gemeinkostenwertanalyse, ab Mitte der 80er Jahre durch fixe Personalabbauzielsetzungen. Zum Personalabbau trat seit Mitte der 80er Jahre die Reduzierung der Fertigungs- 
tiefe hinzu. Besonders lohnkostenintensive Fertigungen sollten aus der Eigenfertigung herausgenommen und fremdvergeben werden. Die Fertigungstiefenreduzierung beruhte in dieser Phase eher auf groben Schätzungen als auf systematischen Kostenberechnungen.

Auch das vierte Strategieelement, die Internationalisierung der Produktion, hatte seinen eindeutigen Schwerpunkt in der Kostensenkung. Durch den Erwerb ausländischer Produktionsstätten an Standorten mit geringerem Lohnniveau und geringeren Sozialleistungen sollten die Kostenstrukturen des Gesamtkonzerns entlastet und preisgünstigere Automobile am Markt plaziert werden. Die Erschließung neuer Märkte blieb zwar ein zentrales Motiv der Internationalisierung, verlor gegenüber dem Kostenargument jedoch an Gewicht. 1982 ergriff die Unternehmensleitung die Gelegenheit, mit dem staatlichen spanischen Automobilproduzenten SEAT eine Kooperation einzugehen, in deren Zentrum die Produktion von Volkswagen an spanischen Standorten stand. 1986 übernahm Volkswagen schließlich das spanische Unternehmen - mit erheblichen Beihilfen der spanischen Regierung - und gliederte es in den Konzern ein. Erstmals entstanden konzerninterne Strukturen der Parallelproduktion, weil an den spanischen Standorten, anders als in den überseeischen Werken, die technologischen und organisatorischen Möglichkeiten vorhanden waren, auch moderne Volkswagen-Modellreihen zu fertigen.

$\mathrm{Zu}$ einem zweiten Element der Internationalisierung wurde in der zweiten Hälfte der 80er Jahre das venture-Management. Volkswagen schloß sich einer Welle internationaler Kooperationen an, die sich in der Automobilindustrie weltweit auszubreiten begann. Die beiden Kooperationen, die das Unternehmen in den 80er Jahren einging, dienten nicht der Erringung einer marktbeherrschenden Stellung, sondern waren Ausdruck der verschärften internationalen Konkurrenz. Durch Zusammenarbeit mit anderen Unternehmen in wohldefinierten Feldern sollte die Schlagkraft der Kooperationspartner für den Konkurrenzkampf mittels Kostensenkungen, Synergieffekten, Kompetenztransfers oder Risikominimierung gestärkt werden. Volkswagens 1986 vollzogene Kooperation mit Ford in Südamerika, bei der die südamerikanischen Tochtergesellschaften beider Unternehmen in eine gemeinsame Gesellschaft, die Autolatina, zusammengeführt wurden, war eine defensive Schicksalsgemeinschaft mit dem Ziel, das Unternehmensrisiko auf dem seit Jahren krisengeschüttelten südamerikanischen Markt für beide Kooperationspartner zu reduzieren und Synergieeffekte in der Produktion zu erzielen. Die 1987 eingegangene Kooperation mit Toyota hatte andere Motive. Die Produktion eines Toyota-Pick-Ups im Werk Hannover sollte einerseits das chronisch unterausgelastete Werk stabilisieren und andererseits Volkswagen die Möglichkeit vermitteln, Einblick in die Organisationsstrukturen des in den 80er Jahren unbestritten erfolg- 
reichsten Automobilproduzenten zu nehmen. Die Internationalisierung der 80er Jahre wurde abgerundet durch die Errichtung einer Produktionsstätte in Shanghai, die der Erschließung des chinesischen Marktes diente und die einen Brückenkopf in Südostasien bilden sollte.

Das Ensemble aus technologischer Rationalisierung, Volumenstrategie, Kostensenkung und Internationalisierung schuf neue Bedingungen für die industriellen Beziehungen und die Interessenvertretungspolitik. Bei Volkswagen kann der interessante Befund formuliert werden, daß sich unter dem Druck der neuen Bedingungen der Charakter der Interessenvertretungspolitik und der industriellen Beziehungen in einer Form änderten, daß sie ihrerseits Einfluß auf die strategischen Ziele der Unternehmenspolitik auszuüben begannen. Die Interessenvertretung entwickelte in den 80er Jahren in der mühsamen Auseinandersetzung mit den neuen Rahmenbedingungen und den allgemeinen gewerkschaftlichen Diskussionen die Konturen einer gestaltungsorientierten Politik der Beschäftigungssicherung. Die traumatischen Krisenerfahrungen der 70er Jahre, die erneute Krise zu Beginn der 80er Jahre und die langfristigen Beschäftigungsbedrohungen durch die technologische Rationalisierung ließen die Beschäftigungssicherung zum unumstrittenen Hauptproblem der Interessenvertretungspolitik werden. Es kristallisierte sich heraus, daß die Sicherung der Beschäftigung mit den traditionellen Mitteln eines passiven Rationalisierungsschutzes immer weniger gewährleistet werden konnte. Unter der Formel der »sozialen Gestaltbarkeit der Technologie « entwickelte die Interessenvertretung durch die Verknüpfung von Rationalisierungsschutz und Humanisierungspolitik die Konturen einer eigenständigen Gestaltungspolitik, die auf die präventive Gestaltung der Technologie setzte. Die Folgewirkungen der Rationalisierung für Beschäftigung und Arbeitsplatzstruktur sollten kontrolliert und beeinflußt werden. In die technologischen Gestaltungsforderungen mischten sich zunehmend organisationskritische Aspekte, die sich vor allem auf die tayloristische Arbeitsorganisation bezogen und die mit dem alten gewerkschaftlichen Ziel der Mitbestimmung am Arbeitsplatz verknüpft wurden. In der zweiten Hälfte der 80er Jahre entwickelte die Interessenvertretung ein umfassendes Konzept der Organisationsinnovation, das auf die Abschaffung des fordistischen Produktionsmodells zugunsten eines dezentralisierten, prozeßorientierten und enthierarchisierten Organisationsmodells mit breiter Einführung strukturinnovativer Gruppenarbeit abzielte. ${ }^{3}$ Die Interessenvertretung entwickelte sich zu einem Promotor der Innovation (Witte 1973).

3 Strukturinnovative Gruppenarbeit zeichnet sich durch eine hohes Maß an Funktionsintegration und breite Dispositionsspielräume der Gruppe hinsichtlich Personal-, Zeit- und Leistungsregulierung aus (Schumann, Gerst 1996). 
Die Internationalisierung gewann als Feld der Beschäftigungspolitik einen immer größeren Einfluß, als mit der Akquisition SEAT's im Konzern erstmals umfassendere technologische Möglichkeiten einer kostenorientierten Standortpolitik geschaffen worden waren. Von diesem Zeitpunkt an wurde die Verteilung von Produkten, Produktionsumfängen, Investitionen und Beschäftigung zu einem integrierten Bestandteil der Verhandlungsprozesse zwischen Unternehmensleitung und Interessenvertretung. Der Interessenvertretung ging es in diesen vehement geführten Verteilungskonflikten mit dem Vorstand reaktiv um die Erhaltung der deutschen Standorte, nicht um ihren weiteren Ausbau. Parallel dazu entwickelte die deutsche Interessenvertretung gemeinam mit den Interessenvertretungen der spanischen Standorte eine neue Form internationaler Gewerkschaftsarbeit, die auf der aktiven Kooperation der europäischen Interessenvertretungen des Konzerns beruhte, mit deren Hilfe die bedrohlichen Auswirkungen der Standortkonkurrenz durch solidarisches Handeln abgefedert werden sollten. Diese Bemühungen mündeten 1990 in die Gründung eines europäischen Konzernbetriebsrats (Euro-KBR), dessen Funktionslogik die solidarische Verteilung von Chancen und Risiken zwischen den Standorten war (Mertens 1994). Es gelang der Interessenvertretung durch starken Druck und durch die Internationalisierung der Gewerkschaftspolitik die drohenden Gefahren einer Lohn- und Sozialkonkurrenz zwischen den Standorten abzuwenden. Eine rein kostenorientierte Standortpolitik hätte von der Unternehmensleitung nur um den Preis der Aufgabe der kooperativen Beziehungen durchgesetzt werden können. Eine Zerreißprobe der industriellen Beziehungen fand nicht statt, weil die Unternehmensleitung die Forderungen der Interessenvertretung in ihre Internationalisierungsstrategie einband. Zwar wurden Produktionsumfänge nach Spanien verlagert, doch geschah dies innerhalb der Wachstumsspielräume, die der Automobilboom in den späten 80er Jahren eröffnete. Auch Kostenvergleiche wurden zwischen den Standorten vorgenommen, doch waren zu keinem Zeitpunkt die Kostenstrukturen die einzige Steuerungsgröße für Standortentscheidungen. ${ }^{4}$

Die Veränderungen der Interessenvertretungspolitik zogen veränderte Formen der Interaktion in den industriellen Beziehungen nach sich. Die Interessenvertretung erhob neue gestaltungspolitische Ansprüche, die in den al-

4 Ein wichtiges Beispiel dafür war die Schließung des US-Werkes Westmoreland in den Jahren 1986/87. In dieser Phase hatte eine kleinere konjunkturelle Schwäche eingesetzt, die erhebliche Überkapazitäten in der Golf-Klasse an den Standorten Wolfsburg, Brüssel und Westmoreland aufdeckte. Nach Kostenkriterien hätte die Produktion im nur zu etwa 50\% ausgelasteten Werk Westmoreland erhöht werden, die Produktion in Brüssel gehalten und in Wolfsburg reduziert werden müssen, selbst unter Einbeziehung der personellen Folgekosten einer Produktionsreduzierung in Wolfsburg. Statt dessen wurde das USWerk geschlossen, die Belieferung des US-Marktes vom mexikanischen Werk in Puebla gestartet und die Produktion in Wolfsburg auf alter Höhe erhalten. 
ten fordistischen Rationalisierungskompromiß nicht mehr einzufügen waren. Zwar verließ sie den kooperativen Produktivitätspakt mit der Unternehmensleitung nicht grundsätzlich, doch versuchte sie, innerhalb dieses Rahmens stärkere Gestaltungsrechte und neue Gestaltungsebenen wie den Euro-KBR durchzusetzen. Das herausragende Beispiel für die Erweiterung der Mitbestimmungschancen innerhalb des gegebenen kooperativen Rahmens war der Techniktarifvertrag von 1987, der bessere Informations- und Mitspracherechte bei der Technologiewahl im Rahmen der Planungsverfahren garantierte. Insgesamt kann von einer Ausdehnung mitbestimmungsrelevanter Regelungsbereiche gesprochen werden. Beschäftigungspolitik, Kostensenkungspolitik, Organisationspolitik und Internationalisierungspolitik wurden gleichermaßen Gegenstand der industriellen Beziehungen. Damit gewannen die industriellen Beziehungen einen direkten Einfluß auf unternehmensstrategische Entscheidungen, die vormals in der unumstrittenen Prärogative des Managements gelegen hatten.

Insgesamt betrachtet trugen die vier genannten strategischen Vorgaben der Unternehmensleitung zur Konservierung des fordistischen Modells bei. Volumenstrategie, technologische Rationalisierung, Kostenreduzierung und Internationalisierung zielten auf eine flexible Optimierung des fordistischen Modells, nicht auf seine Überwindung. Weder gab es nachhaltige Veränderungen auf den unterschiedlichen Ebenen der Organisation noch wurden prinzipiell neue Managementkonzepte eingeführt. Einzig der sporadische und an die tayloristische Organisation der Arbeit angelagerte Einsatz von Qualitätszirkeln zeugte von ersten Ansätzen neuer Organisationsformen. Die neuen Technologien wurden in die alten Strukturen und Prozesse eingepaßt. Die gravierendsten Veränderungen des Modells gab es noch auf dem Feld der Internationalisierung mit den durch die technologischen Möglichkeiten und die industriellen Beziehungen gedämpften neuen Formen einer kostenorientierten Standortpolitik und dem venture-management internationaler Kooperationen. Die Internationalisierung wurde zu einem zentralen Element der Kosten- und Konkurrenzstrategie des Unternehmens. Die Konzentration vollzog sich durch die Akquisition SEAT's in erster Linie als Internationalisierung, zumal die fordistische Konzentrationsstrategie vertikaler Integration durch die Bemühungen zur Fertigungstiefenreduzierung von Seiten des Managements unter Druck geriet.

Gegen Ende der 80er Jahre wurden erstmals auch von der Unternehmensleitung grundlegendere Konzepte eines Organisationswandels ventiliert, einige Zeit nachdem die Interessenvertretung ihre Vorstellungen organisatorischer Innovationen entwickelt hatte. Die Unternehmenskonzepte hatten jedoch nicht die Tiefe und Breite der Vorstellungen der Interessenvertretung. Sie beruhten auf den beiden Pfeilern Gruppenarbeit und cost-centerStrukturen. Beide Elemente sollten einen Prozeß der Dezentralisierung von 
Kompetenzen und Verantwortlichkeiten in Gang bringen. Die in der Folge zwischen Unternehmensleitung und Interessenvertretung geführte Auseinandersetzung über Gruppenarbeit, in der die Unternehmensleitung für konservative Gruppenkonzepte plädierte, ließ allerdings wenig Zweifel daran, daß die Unternehmenskonzepte auf die Integration der neuen Elemente in die bestehende Organisation und nicht auf die Überwindung des fordistischen Modells abzielten.

Zu Beginn der 90er Jahre hat sich der Strukturwandel - oder präziser: die Diskussion über Strukturwandel - bei Volkswagen beschleunigt. Dazu trug zum einen das offensichtliche Scheitern des bis dahin verfolgten Strategieensembles bei. Die Kosten- und Ertragssituation des Unternehmens hatte sich trotz des langanhaltenden Booms nicht nur nicht verbessert, sondern im Gegenteil erheblich verschärft. Der Verfall des operativen Ergebnisses signalisierte wachsende Fixkostenprobleme, die ab 1991 zu einem negativen operativen Ergebnis im reinen Automobilgeschäft und zu break-evenWerten von weit über 100\% führten. Zum anderen hatte sich mit Erscheinen der MIT-Studie über die schlanke Produktion (Womack u.a. 1990) und mit der internationalen Managementdiskussion auch im Top-Management Volkswagens die Überzeugung Bahn gebrochen, daß im internationalen Konkurrenzkampf grundsätzlichere Organisationsänderungen notwendig waren, wollte das Unternehmen gegen die schlanken japanischen Produzenten bestehen können.

Auf Betriebs- und Arbeitsorganisationsebene wurden schlanke Konzepte in zwei Formen implementiert. Einerseits wurde in zwei greenfield-plants, den Werken Sachsen und Martorell, schlanke Strukturen nach dem Vorbild europäischer transplants japanischer Automobilunternehmen kopiert. Teamkonzepte, dezentrale Strukturen und flexible Logistikkonzepte wurden mit dichten Hierarchien und umfassenden Fließsystemen gekoppelt. (Mickler 1996; allgemein: Garrahan, Stewart 1992). Diese schlanken Produktionsformen waren flexibilisierte Varianten einer Massenproduktion, die von den Arbeitsteilungsprinzipien der tayloristischen Organisation nur wenig verloren hatten. Andererseits wurden in den westdeutschen Werken einzelne Elemente schlanker Konzepte eingeführt oder forciert. Dazu gehörten in erster Linie der kontinuierliche Verbesserungsprozeß (KVP), die Gruppenarbeit, die Neuordnung der Meisterorganisation, die Reorganisation der Werkstrukturen und die Einführung einer just-in-time-Logistik (jit). Der auf workshops beruhende KVP sollte durch direkte Beteiligung der Produzenten die wertschöpfenden Tätigkeiten optimieren und die nichtwertschöpfenden Tätigkeiten minimieren, die neue Meisterorganisation zielte auf eine Rollenredefinition der Meister als Coaches und Moderatoren, die neue Werkorganisation stärkte, als Beitrag zur Dezentralisierung, die Stellung der Werke und der Werksleiter gegenüber den zentralen Funk- 
tionen und die jit sollte die aufgrund der komplexen Teile- und Variantenstruktur hohe Pufferbildung durch eine Logistik nach dem Hol-Prinzip minimieren. Diese Instrumente gingen jeweils durch den »Filter« der industriellen Beziehungen und wurden von der Interessenvertretung soweit als möglich mit ihren strukturinnovativen Vorstellungen angereichert. Grundlegende Konfliktpotentiale, beispielsweise über die Ausrichtung der Gruppenarbeit, konnten dabei zwar nicht ausgeräumt werden, doch wurden die Konzepte durchgängig zumindest in Projektform installiert. Dabei bewiesen die Einzelkonzepte in den westdeutschen Werken ein weit größeres Potential für strukturinnovative Überwindungen des Taylorismus als die schlanken Konzepte nach transplant-Vorbildern.

Auf der Unternehmensebene gab es zwei Reformprojekte, die für den Formwandel der Internationalisierung von großer Bedeutung waren. Die Reorganisation der Konzernstrukturen dezentralisierte die einzelnen Marken und Regionen und führte sie in einer matrixförmigen Konzernorganisationsstruktur mit den Funktionen zusammen. »Alle Macht den Marken«, so lautet die dem damaligen Vorstandsvorsitzenden Carl H. Hahn zugeschriebene Losung, die eine konzerninterne Markenkonkurrenz stimulieren sollte. Die in der Parallelproduktion angelegte Konkurrenz der Standorte wurde durch eine Konkurrenz der Marken innerhalb der Organisation und am Markt flankiert und ausgeweitet. Die Dezentralisierung betraf auch die Entwicklung und Konstruktion der Produkte. Beispielsweise unterschieden sich Audi-Produkte von Volkswagen-Produkten durch die Art des Motoreneinbaus.

Das zweite Reformprojekt war die Neuordnung des Produktentstehungsprozesses (PEP). Der PEP sollte nach dem Vorbild des japanischen simultanenous engineering als funktionsübergreifender Prozeß gestaltet werden. Die fordistische Funktionenhierachie zwischen Konstruktion, Fertigung und Vertrieb sollte zugunsten eines integrierten Prozesses abgelöst werden, der sowohl die Funktionen als auch bestimmte Zulieferer direkt einband. Die Voraussetzungen für die Integration von Lieferanten und die Übernahme von Entwicklungsleistungen durch Lieferanten waren die Aufspaltung der Entwicklungs- und Produktionsverantwortung in Module, komplexe Baueinheiten des Automobils, die jeweils von einem Lieferanten entwickelt wurden sowie die Existenz ausreichender Entwicklungskompetenz beim Lieferanten. Angestrebt wurde sowohl ein schnittstellenübergreifender Informations- und Kommunikationsfluß als auch eine Reduzierung der Entwicklungstiefe, um die eigenen Entwicklungsressourcen auf die verbliebenen Restkompetenzen bündeln zu können und die Entwicklungsdynamik zu beschleunigen.

Die Neuordnung des PEP war zugleich ein Angriff auf die fordistischen Formen vertikaler Integration, der weit über die ersten Schritte der Ferti- 
gungstiefenreduzierung der 80er Jahre hinausging. Die Beschaffungsentscheidungen (neudeutsch: Sourcingentscheidungen) wurden systematisch in den Entwicklungsprozeß verlagert und bezogen sich auf Entwicklungen und Fertigungen. Dabei stand prinzipiell der gesamte Bereich der Komponentenfertigung für die Sourcingentscheidungen zur Disposition. Die Zulieferer sollten auf der Grundlage wachsender Vertrauensbeziehungen in Entwicklung und Logistik integriert werden. Es enstand das Bild vernetzter Strukturen mit geringerer Fertigungs- und Entwicklungstiefe des Finalisten. Die internen Komponentenfertigungen drohten in diesem Szenario von externen Konkurrenten überrollt zu werden, weil sie erstens unmittelbar der Konkurrenz externer Anbieter ausgesetzt wurden, zweitens keine Eingriffsrechte in den PEP hatten und drittens als zentral dominierte Einheiten über keine eigenen Entwicklungsleistungen verfügten, die sie mit externen Anbietern hätte konkurrieren lassen können. Die alte Konzentrationsform der vertikalen Integration sollte durch eine netzwerkartige Integration entlang der Produktionskette ersetzt werden, in der die interne Komponentenfertigung keinen systematischen Platz mehr hatte.

Die in den ersten Jahren des neuen Jahrzehnts ventilierten Projekte stellten erstmals wesentliche Elemente des fordistischen Produktionsmodells grundsätzlich in Frage. Doch verlief ihre Einführung zu langsam, als daß sie in kürzerer Frist einen grundlegenden Wandel hätten bewirken können. Es regten sich zu viele innerorganisatorischen Widerstände und Blockaden, und der Nachdruck, mit dem die Unternehmensleitung die Projekte vorantrieb, reichte nicht aus, um die Widerstände schnell oder überhaupt zu überwinden. So bot Volkswagen am Ende des langen Automobilbooms der 80er und frühen 90er Jahre das Bild eines organisatorischen Torsos, und es kann mit Blick auf die vergeblichen und unzureichenden Bemühungen zum Organisationswandel von den für das Unternehmen »verlorenen 80er Jahren« gesprochen werden (Jürgens, Naschold 1994).

Einzig die Internationalisierung war erneut aggressiv vorangetrieben worden. Das Unternehmen nutzte die sich nach dem Zusammenbruch der realsozialistischen Ordnung in den mittel- und osteuropäischen Staaten bietenden Internationalisierungschancen durch den Kauf des tschechoslowakischen Automobilproduzenten Skoda, den Erwerb einer Produktionsstätte in Bratislava und den Aufbau neuer Produktionskapazitäten in Ostdeutschland. Galten diese Projekte immer auch der Erschließung neuer Märkte, so hatten insbesondere für die Skoda-Akquisition die standort- und kostenpolitischen Vorteile hoher Qualifikationsstandards mit niedrigen Löhnen und Sozialleistungen und akzeptabler Infrastruktur den Ausschlag für das Projekt gegeben. Die osteuropäische Internationalisierung war ein weiterer Schritt auf dem Weg zu einer kostenorientierten Standortpolitik. Doch auch in dieser Internationalisierungsphase trugen die vom westdeutschen Ge- 
samtbetriebsrat lancierten Politisierungsversuche der Standortentscheidungen Früchte. Es gelang der Interessenvertretung, für ihre Zustimmung zur weiteren Internationalisierung noch 1990 eine Vereinbarung über die Standort- und Beschäftigungssicherung mit der Unternehmensleitung abzuschließen, in der sich Unternehmensleitung und die Interessenvertretung gleichermaßen zur Standort- und Beschäftigungssicherung und zur Steigerung der Wettbewerbsfähigkeit des Unternehmens bekannten. Wachstumsspielräume sollten für die westdeutschen Standorte ebenso genutzt werden wie Effizienzsteigerungsspielräume. Die Vereinbarung schrieb einen Wachstums- und Modernisierungspakt zwischen Unternehmensleitung und Interessenvertretung fest. Mit der Internationalisierung veränderten sich die industriellen Beziehungen in Richtung einer Modernisierungskoalition, die jedoch durch die unzureichenden Modernisierungsanstrengungen der Unternehmensleitung gehemmt wurde.

Auch am Ende des langen Booms war die kostenorientierte Standortpolitik noch kein umfassendes Steuerungsprinzip. Dies lag jedoch nicht nur an der Politisierung der Standortentscheidung, sondern auch an der Persistenz multinationaler Strukturen. Die alten technologischen und organisatorischen Lücken zwischen der VW AG als Konzernmutter und den überseeischen Töchtern bestanden noch immer, die Töchter agierten weiterhin relativ autonom und die kostenorientierte Standortpolitik beschränkte sich auf eher geringe Möglichkeiten konzerninterner Parallelproduktion. Von einem umfassenden Strukturwandel der Internationalisierung kann daher für diesen Zeitraum weniger gesprochen werden als von einem Wandel der strategischen Motive.

\section{Auf dem Weg zum globalen Produktionsnetzwerk}

Der dramatische Kriseneinbruch des Jahres 1993 bildete einen tiefen Einschnitt in der Unternehmensentwicklung. Die Unternehmensleitung unter dem Anfang 1993 berufenen neuen Vorstandsvorsitzenden Ferdinand Piëch schlug zwei Krisenstrategien ein. Die erste Strategie war ein kurzfristiges Kostenmanagement, in dessen Verlauf Produktion und Investitionen, aber auch die Beschäftigung teilweise drastisch zurückgefahren wurden. Die zweite Strategie war ein Programm der langfristigen Organisationstransformation, für das der neue Vorstandsvorsitzende sehr intensiv warb. Das Piëchsche Transformationsmanagement hatte seine Schwerpunkte in der Dezentralisierung, Prozeßorientierung und Vernetzung der Strukturen.

Die gesamte Wertschöpfungskette sollte als funktions- und unternehmensübergreifender Prozeß $\beta$ reorganisiert werden. Die funktionalen Schnittstellen innerhalb des Unternehmens und die Unternehmensschnittstellen zwischen Volkswagen und den Lieferanten sollten abgebaut werden. In den 
Zulieferbeziehungen sollten Formen längerfristiger Kooperation die reinen Marktbeziehungen wenn nicht ganz ersetzen, so doch wesentlich ergänzen. Zulieferer sollten in die Entwicklung, die Logistik und die Fertigung des Finalisten integriert werden. Zugleich sollten globalisierte Sourcingabläufe standortbedingte Leistungsdifferenzen von Zulieferern erschließen. Die Strukturen des Unternehmens sollten in Kreisen, Gruppen und Netzwerken organisiert werden. Die Abflachung von Hierarchien, die Dezentralisierung von Kompetenzen und die Abschwächung der Funktionen sollten prozeßorientierte Abläufe stärken. Qualitäts- und Kundenorientierung sollten als durchgängige Orientierungen in allen Prozessen verankert werden. Die weichen Faktoren der Unternehmenskultur gewannen neben den harten Faktoren der Organisationsstrukturen eine neue Bedeutung. Beabsichtigt war der Übergang von der »Zweckgemeinschaft« Unternehmen zu einer »Sinngemeinschaft« mit einer ausgeprägten Beteiligungskultur des Einzelnen, was diesem eine entsprechend höhere und intensivere Arbeitsleistung abverlangt.

Der internationale Konzernverbund sollte zu einem vernetzten »Verbund für Wertschöpfung « ausgestaltet werden, der sowohl Dezentralisierungsals auch Synergiepotentiale auszuschöpfen versprach. Auf der einen Seite sollten die entstandenen Formen der Standortkonkurrenz systematisiert und ausgebaut werden. Auf der anderen Seite sollte über die Einführung weniger markenübergreifender Produktionsplattformen die Teilevielfalt reduziert und die Produktvielfalt der Endprodukte erhöht werden. Plattformen waren standardisierte Elemente, die modell- und markenübergreifend in allen Fahrzeugen einer Plattform eingebaut wurden und die für den Kunden nicht unmittelbar sichtbar waren wie beispielsweise Achsen, Böden, Heizung, Aggregate oder Sitzsysteme. Die Produktvielfalt wurde durch die Außenhüte erzielt, die den jeweiligen Plattformen überzustülpen waren. Die Plattformstrategie sollte sowohl die Skaleneffekte der Massenproduktion mit gestiegener Produktvielfalt verbinden als auch die konzernweite Produktionsflexibilität zwischen den Standorten erhöhen. Die konzernweite Plattformstrategie mußte $\mathrm{zu}$ einer technologischen und organisatorischen Angleichung der Modernitätsgrade zwischen den Standorten führen.

Die zentralen Elemente des Piëchschen Transformationskonzeptes deckten sich weitgehend mit den Vorstellungen der Interessenvertretung. Die Dezentralisierung und Verlagerung von Kompetenzen nach unten, die ProzeBorientierung und die Abschwächung der Funktionen, die Entbürokratisierung und die breite Einführung von Gruppenarbeitsstrukturen, dies waren seit Mitte der 80er Jahren die Kernpunkte des interessenvertretungspolitischen Organisationskonzeptes, das mit der alten Unternehmensleitung nicht hatte durchgesetzt werden können. Dies galt bemerkenswerterweise auch für die Gleichteilepolitik. Die Interessenvertretung hatte seit Ende der 70er 
Jahre eine Gleichteilestrategie gefordert und den unter Hahn betriebenen markenspezifischen Differenzierungen sehr ablehnend gegenübergestanden. Aufgrund der breiten Schnittmenge beider Konzepte zeichnete sich schnell eine Modernisierungskoalition zwischen Unternehmensleitung und Interessenvertretung ab, in der beide Koalitionspartner neue Organisationskonzepte gegen interne Widerstände und Blockaden durchsetzen wollten. Doch die Koalition beschränkte sich nicht allein auf die Modernisierung, sie entwickelte sich zugleich zu einer Beschäftigungskoalition. Beide Aspekte wurden in den bahnbrechenden Tarifverträgen zur Sicherung der Standorte und der Beschäftigung der Jahre 1993 und 1995 auch formal fixiert. In diesen Tarifverträgen verpflichteten sich beide Tarifpartner, Unternehmensleitung und IG Metall, zur Standort- und Beschäftigungssicherung und zur Verbesserung der Wettbewerbsfähigkeit des Unternehmens (Hartz 1994 und 1996, Promberger u.a. 1996). Anders als die Vereinbarung zur Standortsicherung des Jahres 1990 waren die Tarifverträge keine auf Wachstumsprozesse beruhenden passiven Willensbekundungen, sondern allgemeingültige aktive Verpflichtungen, die mit den konkreten Maßnahmen der drastischen Arbeitszeitverkürzung ohne Lohnausgleich - der vierTage-Woche - und weiteren innovativen Arbeitszeitkonzepten wie der Stafettenregelung und der Blockwahl ausgefüllt wurden. Beschäftigungssicherung gegen Leistungssteigerungen und Innovationen, so lautete die Tauschformel der Tarifverträge, die zugleich für die Interessenvertretung eine Einladung zur Einbringung eigener Innovationskonzepte war.

Der Verlauf der Organisationstransformation in den Jahren ab 1993 zeigte, $\mathrm{da} ß$ die Interessenvertretung diese Möglichkeit offensiv nutzte. Allerdings lief der Reorganisationsprozeß in einigen Bereichen sehr zäh an. Das Stiefkind der Reorganisation war bis zum Ende des Untersuchungszeitraums zweifelsohne die Arbeitsorganisation. Von der angekündigten breitflächigen Umsetzung der Gruppenarbeit war zunächst wenig zu spüren. Im Gegenteil, die Gruppenarbeitsprojekte stagnierten eher. Lediglich im Montagebereich der 1995 in Wolfsburg erneut aufgenommenen Polo-Produktion wurde Gruppenarbeit als durchgängiges Organisationsprinzip eingeführt, doch auch in diesem kleinen Produktionssegment nur in sehr restriktiven Formen. Statt dessen konzentrierten sich die arbeitsorganisatorischen Bemühungen der Unternehmensleitung ganz auf die Revitalisierung des KVP (kontinuierlicher Verbesserungsprozeß), der unter dem im Frühjahr 1993 berufenen neuen Vorstand für Produktionsoptimierung und Beschaffung, José Ignacio López de Arriortúa, als $\mathrm{KVP}^{2}$ auf breiter Front eingeführt wurde. $^{5}$ Nach anfänglichen Erfolgen zeigte der neue KVP in der Mitte der

5 Der Prozeß wurde nun als $\mathrm{KVP}^{2}$ bezeichnet, weil über ein »Schneeballsystem« kontinu- 
90er Jahre immer deutlichere Ermüdungserscheinungen. Die erhofften schnellen Rationalisierungserfolge erwiesen sich als wenig nachhaltig, weil der KVP als »Ersatzstrategie« (Schumann 1996, 255) für umfangreichere Reformen der Arbeitsorganisation bloß neben die bestehenden und noch immer vom Taylorismus beherrschten Strukturen gestellt und dementsprechend nicht durch Gruppenarbeit organisatorisch abgestützt wurde. Ende 1995 galt auch offiziell dieser Weg des geringsten Widerstands als gescheitert. Die Arbeitsorganisation, bis in die 90er Jahre der Kern der Reorganisationsbemühungen im Unternehmen, wurde zu einem bloßen Anhängsel weiterer Reformen.

Eine ganz andere Bilanz ist bei der Reorganisation der sourcing-Prozesse und der Einführung vernetzter Zulieferstrukturen zu ziehen. Die in diesem Bereich entstandenen vernetzten Strukturen zwischen internen Zulieferern und Montagewerken und die Reorganisation der internen Zulieferer zu Systemlieferanten wurden zum Vorbild und zum Motor für die Reorganisation der gesamten Konzernstrukturen. In Verbindung mit der konsequent umgesetzten Plattformstrategie wurden die Grundlagen für den Umbau des multinationalen Konzerns zu einem global atmenden Produktionsnetzwerk gelegt.

Die Reorganisation der sourcing-Prozesse und die Vernetzung der Zulieferbeziehungen erfolgte in drei Schritten. Im Verlauf dieser Schritte wandelte sich nicht nur das Verhältnis von Abnehmer und Zulieferer, sondern auch die Stellung der internen Komponentenfertigung, das gesamte Bild des Unternehmens- und Betriebszusammenhanges und die Rolle der industriellen Beziehungen im Rationalisierungsprozeß. Der erste Schritt war die Neuordnung des Sourcing-Verfahrens. Nach den Piëchschen Vorgaben sollten die Zulieferbeziehungen integriert und internationalisiert werden. Beide Forderungen wurden in dem einvernehmlich mit dem Betriebsrat geregelten und in einer Betriebsvereinbarung festgeschriebenen Standardablauf des forward- und global-sourcing vereint (vgl. dazu Gehrke 1997). Forward-sourcing meint die frühzeitige Einbindung von Lieferanten in den Entwicklungsprozeß neuer Produkte, global-sourcing die kontinuierliche Suche nach den kostengünstigsten Lieferanten bei laufenden Modellen. Der Standardablauf systematisierte den Beschaffungsprozeß und sicherte die vom Betriebsrat seit langem geforderte Beteiligung im Produktentstehungsprozeß zu. Das Verfahren sah einen vierstufigen Ablauf von der Vorbereitung, Ausschreibung und Verhandlung bis zur Entscheidung vor. Es systematisierte jedoch nicht nur den Ablauf, sondern enthielt zwei weitere wich-

ierliche Anschlußworkshops durchgeführt werden und der KVP entlang der gesamten Wertschöpfungskette, also auch bei den Zulieferern, durchgeführt werden sollte. Dadurch sollte eine potenzierte Wirkung entstehen. 
tige Elemente. Zum einen wurden den internen Komponentenfertigern im Rahmen des sogenannten »last call« Sonderkonditionen eingeräumt. Sie hatten die Möglichkeit, bei Preisüberschreitung im Vergleich zu externen Anbietern interne Optimierungsschleifen zu durchlaufen, um mit den externen Anbietern gleichziehen oder sie sogar unterbieten zu können. Die internen Komponentenfertigungen bekamen damit eine zweite Chance für ein überarbeitetes Angebot. Dieses Verfahren garantierte zugleich ein kontinuierliches benchmarking der internen Lieferanten nach den jeweils besten Marktstandards, was auf die dort Beschäftigten natürlich auch einen gehörigen Druck ausübt. Zum anderen wurde der Betriebsrat der Komponentenfertigung fest in den Sourcingprozeß integriert. Nicht nur wurde er auf jeder der Verfahrensstufen ausführlich informiert, er wurde auch aktiv in den Optimierungsprozeß der Eigenfertigung einbezogen. In diesem Prozeß übernahm der Betriebsrat originäre Managementfunktionen eines Kosten-, Produktivitäts- und Innovationsmanagements und wurde $\mathrm{zu}$ einem CoManager der Optimierung (Girndt 1997, 41-50). Die Betriebsvereinbarung enthielt darüber hinaus mit den jährlich abzuhaltenden Werksymposien ein neues Kommunikationsforum zwischen Betriebsrat, Werkmanagement und Unternehmensleitung über strategische Fragen der Produkt-, Werks- und Investitionsentwicklung, auf denen die Interessenvertretung umfassend informiert wurde und eigene strategische Überlegungen in der Prozeß der Strategiebildung einspeisen konnte. In einer von der Interessenvertretung geforderten und 1995 vereinbarten Protokollnotiz wurden die Sonderchancen der internen Fertigung präzisiert und ausgeweitet. Der internen Fertigung wurde - unter Wahrung der Grundbedingung der Wettbewerbsfähigkeit - dadurch Priorität zugesprochen, daß die Beschäftigungssicherung als ausdrückliches Ziel in der Vereinbarung festgeschrieben und in den Prozeß verankert wurde.

Der zweite Schritt der Reorganisation war die Integration der Zulieferer in die Unternehmensstrukturen des Finalisten VW. Unter dem Begriff des insourcing wurden Formen räumlicher Integration der Zulieferer diskutiert. Ansiedlungen am und auf dem Werksgelände, die Endmontage der Module vor Ort und die Anlieferung der Module bis unmittelbar zum Einbau in der Endmontage sollten nicht nur die Paßgenauigkeit der Anlieferung optimieren, sondern auch Arbeitsplätze an den Standorten der VW AG sichern. Stellten schon diese Formen räumlicher Integration einschneidende Veränderungen für die klassischen Betriebsstrukturen dar, weil Lager oder Fertigungen der Zulieferer auf dem Werkgelände plaziert wurden, so war die Idee, auch die Endmontage der Module in die Fahrzeuge durch Zulieferer ausführen zu lassen, mit dem traditionellen Verständnis einer technologisch-organisatorischen Einheit des Betriebes nicht mehr verträglich. Die Modullieferanten sollten nicht nur in den Entwicklungs- und Logistikpro- 
zeß, sondern auch in den Fertigungsprozeß des Endabnehmers VW integriert werden. Unter dem Titel fraktale Fabrik wurden bei Volkswagen noch weitergehende Integrationsformen diskutiert, die die Übernahme weiterer zentraler Funktionen des Automobilbaus, beispielsweise der Lackiererei, in die Produktions-, Personal- und Investitionsverantwortung von Lieferanten vorsah. Von einer modularen Fabrik wurde für den Fall gesprochen, daß auch die internen Prozesse in kleine, selbstorganisierende und selbstregulierende Einheiten dezentralisiert sind.

In der realen Umsetzung wurden Mitte der 90er Jahre auf breiter Front »fraktale Projekte « gestartet, die vornehmlich an den europäischen Auslandsstandorten, aber auch im Inland anliefen. Unter den Projekten ragten die Übernahme der Lackierereifunktion in einem SEAT-Werk durch einen Zulieferer und die umfangreiche räumliche Ansiedlung von Zulieferern mit Montagekompetenzen auf dem Werkgelände bei Skoda hinaus. Den vorläufigen Höhepunkt und das vorläufige Ende des Fraktalisierungsbooms stellte das neue LKWWerk der VW do Brasil in Resende dar. Das Werk war 1996, nach der Auflösung der mit Ford betriebenen gemeinsamen Gesellschaft Autolatina, wegen steigenden Kapazitätsbedarfs in Bau gegangen und sollte der Präzedenzfall einer umfassenden Modularisierung im Konzern werden. Während bei VW noch die Gesamtkoordination und die Qualitätssicherung verblieb, sollten alle anderen Funktionen von Zulieferern mit eigener Produktions-, Personal- und Investitionsverantwortung übernommen werden. Daß das in Resende verfolgte Konzept der Konzernleitung letztlich nicht aufging, sondern VW weit mehr Funktionen selber übernehmen mußte, als dies in den ursprünglichen Planungen vorgesehen war, dürfte vorrangig zwei Gründen zu verdanken sein. Einerseits entsprachen die weitgehenden Vorstellungen des Unternehmens offensichtlich nicht den Möglichkeiten und den Interessen der Zulieferer. Denn die Übernahme zentraler Automobilbaufunktionen erforderte auf Seiten der Zulieferer nicht nur umfangreiche Kompetenzen, sondern auch den Einsatz erheblicher finanzieller Ressourcen vor allem durch die Investitionsverantwortung, die die Zulieferer zu übernehmen hatten. Zudem entstanden durch die Investitionen in die Fabriken des Abnehmers neue Abhängigkeiten der Zulieferer vom Endproduzenten, sowohl im Hinblick auf den Markterfolg des Finalisten als auch im Hinblick auf die Abhängigkeit von seinen weiteren strategischen Standortentscheidungen. Aufgrund des hohen finanziellen Risikos und der hohen Abhängigkeiten war die Fraktalisierung für die Zulieferer bislang zu wenig attraktiv, um sich in ihrer radikalen Form durchsetzen zu können, und auch große globale Finalisten wie Volkswagen scheinen nicht die Macht zu haben, Zulieferer zu diesem Schritt zwingen zu können, zumal in diesem Fall die für das Gelingen integrativer und kooperativer Beziehungen zwischen Finalist und Zulieferer notwendige Ressource Vertrauen gefährdet werden würde. 
Andererseits hat die Politik der Interessenvertretung eine ungezügelte Fraktalisierung einzudämmen versucht, weil mit der Auflösung der klassischen Betriebs- und Unternehmensstrukturen in Netzwerke die rechtlichen Grundlagen der Mitbestimmung ausgehebelt zu werden drohten und die Beschäftigung der VW-Kernbelegschaft gefährdet schien. Mit zwei Forderungen, der Festschreibung von Kernkompetenzen des Finalisten VW und der Einführung fester Integrationsgrenzen für die Zulieferer, sollte gleichermaßen die physische Einheit von Unternehmen und Betrieb, die $\mathrm{Zu}$ kunftsfähigkeit des Unternehmens in strategischen Kompetenzen und die Beschäftigung gesichert werden. Die Interessenvertretung verband mit dieser Position keine grundlegende Ablehnung modularer Konzepte. Vielmehr versprach sie sich gerade von der Idee selbstorganisierender und -regulierender Einheiten eine wirkungsungsvolle Form der Dezentralisierung, die bei Volkswagen noch immer nicht hatte eingeführt werden können. Doch beruhte das Konzept der Interessenvertretung weniger auf der netzwerkartigen Integration der externen Zulieferer als auf der Integration der internen Komponentenfertigungen. Über eine interne Fraktalisierung sollte die vertikale Integration in eine vernetzte Integration umgewandelt werden. Diese Entwicklung war jedoch ausgeschlossen ohne den dritten Schritt der Reorganisation, die Umwandlung der internen Komponentenfertiger in vollwertige Systemlieferanten mit durchgängigen Kompetenzen von der Modulentwicklung bis zum Moduleinbau. Dieser Schritt war für die weitere Reform der Werks- und Konzernstrukturen von kaum zu überschätzender Bedeutung, weil er der Motor eines konzernweiten Dezentralisierungprozesses war, in den die gesamten Organisationsstrukturen hineingezogen wurden. Der Schrittmacher der Dezentralisierung war der Betriebsrat des Werkes Braunschweig. Der Betriebsrat hatte schon zu Beginn der 90er Jahre die großen Gefahren erkannt, die sich aus den neuen Zulieferkonzepten für die internen Komponentenfertigungen ergeben mußten, insbesondere für ein Werk wie Braunschweig, dessen gesamte Produktpalette auch mühelos von außen bezogen werden konnte. Der Betriebsrat war sich darüber im klaren, daß das Werk mittelfristig als von zentralen Funktionen abhängiger Teilelieferant gegenüber modernen Modullieferanten mit eigenen Entwicklungskompetenzen nicht würde bestehen können. Anders als die externe Konkurrenz konnte das Werk keine eigenen Entwicklungen vornehmen und deshalb weder analog der großen Lieferanten in die Entwicklungsprozesse integriert werden noch mit der notwendigen Dynamik und im notwendigen Umfang komplexe Modulentwicklungen vorantreiben. Die internen Komponentenfertiger drohten in das zweite Glied der Zulieferer abzusinken oder ganz vom Markt zu verschwinden. Vor diesem Hintergrund startete der Betriebsrat eine Beschäftigungsinitiative, in deren Zentrum die Reform der Komponentenfertigung stand. Das Ziel war, in der in- 
ternen Fertigung die Entwicklung der externen Konkurrenz nachzuvollziehen. Wenn schon der Konkurrenzkampf nicht zu vermeiden war, so sollte er zumindest mit gleichen Chancen für die internen Fertigungen ausgetragen werden. Der Betriebsrat forderte umfassende eigene Entwicklungskompetenzen für den Standort und eine eigene Logistikverantwortung, um interne jit-Strukturen entwickeln zu können. Im Lauf der folgenden Jahre traten zu diesen Forderungen noch der Anspruch auf eigene Vertriebs-, Investitions-, und Kooperationsverantwortung und schließlich auf die Moduleinbaukompetenz vor Ort hinzu. Braunschweig sollte von den zentralen Funktionen abgenabelt werden und eine durchgängige Prozeßflußverantwortung von der Entwicklung bis zum Einbau von Modulen zuerkannt bekommen. Der Betriebsrat fand als Promotor der Organisationsinnovation einen Verbündeten nicht im Werkmanagament, sondern in der neuen Unternehmensleitung unter Ferdinand Piëch. Es war die Modernisierungskoalition zwischen Betriebsrat und Unternehmensleitung, die die Dezentralisierung der Werkstrukturen einleitete und durchsetzte. Zunächst sollte die Dezentralisierung in der Form eines werkweiten profit-centers Braunschweig vollzogen werden. Doch sowohl Betriebsrat als auch Unternehmensleitung nahmen von diesem Modell schnell wieder Abschied, weil sie mit dem profit-center-Konzept gravierende Nachteile verbanden, die aus der kurzfristigen Profitorientierung und den synergiezerstörenden Konkurrenzformen resultierten, die in einer rein profitorientierten Steuerungsform angelegt waren. Diese Defizite sollten durch das business-unit-Konzept überwunden werden, das neben einer umfassenden Delegation aller funktionalen Kompetenzen auch die Anbindung der units an die strategischen Rahmenvorgaben der Unternehmensspitze vorsah. Die Flexibilitäts- und Effizienzvorteile der Dezentralisierung sollten mit den Synergievorteilen einer integrierten strategischen Ausrichtung verknüpft werden. Damit wurden auch die Voraussetzungen für das Betriebsratskonzept der internen Fraktalisierung, der vernetzten Integration der internen Komponentenfertiger in Entwicklung, Logistik und Fertigung, geschaffen. Am Ende des Untersuchungszeitraums kann festgestellt werden, daß die interne Fraktalisierung deutlich weiter fortgeschritten war als die Fraktalisierung der externen Anbieter, weil die Risiken für die internen Fertigungen deutlich niedriger angesiedelt waren. Vernetzung fand in ihren weitestgehenden Formen als interne Vernetzung statt.

Die unit-Organistion des Komponentenwerkes Braunschweig wurde zum Vorbild für die Ausgestaltung der gesamten Unternehmensorganisation. Das unit-Konzept sollte breitflächig Einzug in die Organisation aller Werke halten, eine Vorstellung die von Unternehmensleitung und Interessenvertretung gemeinsam getragen wurde. Die units sollten durch Einbindung der Funktionen in durchgängige Prozesse die Prozeßorientierung der Organisation gegenüber der funktionalen 
tion gegenüber der funktionalen Ausrichtung stärken. Über den Umweg der unit-Organisation sollte auch die Reform der Arbeitsorganisation revitalisiert werden. Innerhalb der units sollte eine prozeßorientierte und produktbezogene Segmentierung der Fertigungsstrukturen eingeführt werden, die von Teamstrukturen entlang der Prozesse zu tragen war. Darüber hinaus sollten die einzelnen units mit Hilfe moderner Informations- und Kommunikationstechnologien im Konzernverbund global vernetzt werden, so daß die vernetzte Integration zu einer globalen Integration ausgebaut werden konnte.

Doch zur globalen Vernetzung der units trugen nicht nur die neuen Technologien bei, sondern auch und in erster Linie die konsequent umgesetzte Plattformstrategie der Unternehmensleitung, an deren Ende das Bild eines global atmenden Produktionsetzwerkes stand. Die Plattformen erschlossen dem Unternehmen nicht nur Skaleneffekte bei wachsender Produktvielfalt, sondern sie führten auch zu einer technologischen und organisatorischen Angleichung der Modernitätsstandards und zu einer neuen Form der Produktionsflexibilität zwischen den Standorten einer Plattform. Plattformen schufen neue Möglichkeiten, die Produktion in globaler Dimension an die Schwankungen des Marktes anzupassen. Die mit dem Markt atmende Fabrik (Hartz 1996) wurde zu einer produktionstechnologischen Realität, je mehr die Plattformentwicklung und die Produktentwicklung auf Basis der Plattformen voranschritt und je mehr sich die Modernisierungsstandards der Standorte annäherten. Dieser Prozeß vollzog sich, unterstützt von hohen Auslandsinvestitionen zur Modernisierung der osteuropäischen und überseeischen Standorte und zum Aufbau neuer Standorte, rasch und ohne größere Reibungen, so daß am Ende des Untersuchungszeitraums die Plattformstrategie fast vollständig umgesetzt war.

Die Interessenvertretung trug die neue Strategie prinzipiell deshalb mit, weil sie sich davon, ebenso wie die Unternehmensleitung, entscheidende Impulse für die Effizienzsteigerung im Unternehmen erhoffte. Allerdings war für sie die Plattformstrategie ein zweischneidiges Schwert, weil sie neben der Effizienz auch die Standortkonkurrenz weiter stärkte. In einem global atmenden Produktionsnetzwerk wurde die Parallelproduktion zu einem umfassenden Prinzip, und die Produktion konnte zwischen den Standorten einer Plattform beliebig verschoben werden. Es entstand daher die zentrale Frage nach den Kriterien der Standortbelegung in globaler Dimension. Für die Interessenvertretung war klar, daß diese Kriterien sich nicht in kurzfristigen Kostenkalkülen erschöpfen durften, sollten nicht die deutschen Standorte mit ihren noch nicht vollständig bewältigten Strukturproblemen und ihren vergleichsweise hohen Lohn- und Sozialstandards auf das Abstellgleis geschoben werden. Statt dessen sollte das Prinzip einer solidarischen Verteilung von Chancen und Risiken zwischen den Standorten in 
der Tradition des Euro-KBR auch in der globalen Plattformstruktur aufrechterhalten werden. Dazu mußten jedoch die Regelungskompetenzen des europäischen Gremiums auf die globale Konzernebene erweitert werden. Daß diese Erweiterung im Konsens mit der Unternehmensleitung schrittweise durchgeführt werden konnte und im Sommer 1998 zur Bildung eines Welt-KBR führte, ist nicht nur ein deutliches Zeichen für die traditionell kooperativen industriellen Beziehungen im Unternehmen, sondern auch ein Zeichen dafür, daß das Management gewillt war, die Interessenvertretungen und ihre Ziele in die neuen globalen Konzernstrukturen zu integrieren. Insofern kann man von einer gelungenen flexiblen Anpassung der industriellen Beziehungen an die neuen Formen der Internationalisierung sprechen, eine Anpassung, die Volkswagen einmal mehr zum Vorreiter der Entwicklung der industriellen Beziehungen machte.

Das gesamte Produktionssystem und mit ihm die Formen der Internationalisierung befinden sich in einem anhaltenden Prozeß des Wandels, dessen Richtung durch die bereits eingeführten strategischen Konzepte weitgehend vorgezeichnet ist. Plattformstrategie, unit-Organisation und neue Zulieferbeziehungen haben Fixpunkte einer neuen Unternehmensorganisation geschaffen, an deren Ende das global atmende Produktionsnetzwerk mit flachen Hierachien, dezentralisierten Kompetenzen und partizipativen Strukturen stehen soll. In diesem Modell hat die Internationalisierung einen neuen Stellenwert erfahren, weil sie, anders als im fordistischen Modell, zu einem elementaren Bestandteil der flexiblen und auf Massenproduktion ausgelegten Produktions- und Organisationsstrukturen geworden ist. Das global atmende Produktionsnetzwerk ist ein Versuch, die Effizienzvorteile der Massenproduktion mit einer neuartigen Form der Produktionsflexibilität und der Produktvielfalt zu verknüpfen und das in den 70er und 80er Jahren aufgebrochene Rationalisierungsdilemma des Fordismus zu überwinden.

Zwar entwickelte sich mit der Plattformstrategie die Standortkonkurrenz zu einem umfassenden Steuerungsprinzip, doch konnten neue, globale Strukturen der industriellen Beziehungen sowohl im Bereich der Beschaffung als auch und vor allem in Form eines Welt-KBR implementiert werden, die Aspekte der Beschäftigungs- und Standortsicherung in die globalen Entscheidungen einfließen lassen. Auf diese Weise spielt die Beschäftigungssicherung auf der Grundlage der ökonomischen Konkurrenzfähigkeit eine eigenständige Rolle im globalisierten Netzwerk. Die Beschäftigungssicherung geht dabei mit der Ausschöpfung aller Leistungsreserven der Beschäftigten einher, die sowohl mit internen wie mit externen Fertigern konkurrieren müssen. »Druckfreie« Räume der Arbeit schrumpfen durch die umfassende Wettbewerbsorientierung des Modells zusammen.

$\mathrm{Ob}$ das globale Produktionsnetzwerk das fordistische Produktionsmodell als neues best-practice-Modell ablösen und ob es eine ähnliche Verbreitung 
finden wird, ist müßig zu spekulieren. Doch zumindest Eines dürfte sicher sein. Wegen der auf Beschleunigung, Effizienzzunahme und Produktivitätsverbesserung abzielenden Stoßrichtung des neuen Modells wird das Problem der Beschäftigungssicherung wohl ein dauernder und hartnäckiger Begleiter bleiben, solange die Arbeitslosigkeit weiterhin hoch bleibt. Eine ganz andere und an dieser Stelle nicht zu beantwortende Frage ist die ökologische Verträglichkeit des neuen Modells. Hier können Grenzen sowohl hinsichtlich der permanenten Beschleunigung der Produktion als auch hinsichtlich der weiteren Verbreitungsfähigkeit des Produkts Automobil vermutet werden, selbst in seinen Sparversionen eines Drei- oder Zwei-LiterAutos.

\section{Literatur}

Berger, Suzanne; Dore, Ronald (Eds.) (1996): National Diversity and Global Capitalism, Ithaca/London.

Boyer, Robert (1990): The Regulation School. A Critical Introduction, New York.

Boyer, Robert; Durant, Jean-Pierre (1997): After Fordism, Houndmills.

Dunning, John H. (1993): The Globalization of Business. The Challenge of the 1990's, London/New York.

Garrahan, Philip; Stewart, Paul (1992): The Nissan Enigma, Flexibility at Work in a Local Economy, London.

Gehrke, Jörg (1997): Global und Forward Sourcing bei der VW AG, in: Müller, Hans-Erich; Prangenberg, Arno (Hg.), Outsourcing-Management. Handlungsspielräume bei Ausgliederung und Fremdvergabe, Köln, 292-306.

Girndt, Cornelia (1997): Anwälte, Problemlöser, Modernisierer, Gütersloh.

Hartz, Peter (1994): Jeder Arbeitsplatz hat ein Gesicht. Die Volkswagen-Lösung, Ffm.

- (1996): Das atmende Unternehmen. Jeder Arbeitsplatz hat einen Kunden, Ffm.

Herrigel, Gary (1996): Industrial Constructions. The Sources of German Industrial Power, Cambridge 1996.

Hickel, Rudolf (1996): Internationalisierung der Produktion und Globalisierung der Finanzmärkte - Folgen für Arbeit und Gewerkschaften, in: Gewerkschaftliche Monatshefte 11-12, 707-714.

Hollingsworth, J. Rogers; Schmitter, Philippe C.; Streeck, Wolfgang (1994): Capitalism, Sectors, Institutions and Performance, in: dies., Governing Capitalist Economies. Performance and Control in Economic Sectors, New York/Oxford, 3-16.

Hübner, Kurt (1989): Theorie der Regulation. Eine kritische Rekonstruktion eines neuen Ansatzes in der politischen Ökonomie, Berlin.

Joas, Hans (1992): Die Kreativität des Handelns, Ffm.

Jürgens, Ulrich; Naschold, Frieder (1994): Arbeits- und industriepolitische Entwicklungsengpässe der deutschen Industrie in den 90er Jahren, in: Zapf, W.; Dierkes, M. (Hg.), WZBJahrbuch 1994: Institutionenvergleich und Institutionendynamik, Berlin, 239-271.

Kern, Horst (1998): Mitbestimmung und Innovation, Gütersloh.

Koch, Günther (1987): Arbeitnehmer steuern mit. Belegschaftsvertretung bei VW ab 1945, Köln.

Lipietz, Alain (1985): Akkumulation, Krisen und Auswege aus der Krise. Einige methodische Anmerkungen zum Begriff der Regulation, in: Prokla, Heft 58, 109-137.

Lockwood, David (1964): Social Integration and Systems Integration, in: Zollschan, G.; Hirsch, W. (Ed.), Explorations in Social Change, London, 244-257.

Mahnkopf, Birgit (1988), Soziale Grenzen »fordistischer Regulation«, in: dies. (Hg.), Der gewendete Kapitalismus. Kritische Beiträge zur Theorie der Regulation, Münster, 99-143. 
Mertens, Volker (1994): Europaweite Kooperation von Betriebsräten multinationaler Konzerne. Das Beispiel des Volkswagen-Konzerns, Wiesbaden.

Mickler, Otfried; Engelhard, Norbert; Lungwitz, Ralph; Walker, Bettina (1996): Nach der Trabi-Ära: Arbeiten in schlanken Fabriken. Modernisierung der ostdeutschen Automindustrie, Berlin.

Promberger, Markus; Rosdücher, Jörg; Seifert, Hartmut; Trinczek, Rainer (1996): Beschäftigungssicherung durch Arbeitszeitverkürzung. 4-Tage-Woche bei VW und Freischichten im Bergbau: Mehr als zwei Beispiele, Berlin.

Rogers, Joel; Streeck, Wolfgang (Ed.) (1995): Works Councils. Consultation, Representation and Cooperation in Industrial Relations, Chicago.

Schumann, Michael (1996): Gruppenarbeit und Zukunft der Industriearbeit in Deutschland, in: Kißler, Leo (Hg.), Toyotismus in Europa. Schlanke Produktion und Gruppenarbeit in der französischen und deutschen Automobilindustrie, Ffm., 253-262.

Schumann, Michael; Gerst, Detlef (1996): Produktionsarbeit - Bleiben die Entwicklungstrends stabil? in: Jahrbuch Sozialwissenschaftliche Technikberichterstattung 1996, Schwerpunkt: Reorganisation, Berlin, 131-168.

Simons, Rolf, Westermann, Klaus (Hg.) (1997): Standortdebatte und Globalisierung der Wirtschaft, Marburg.

Soskice, David (1994): Innovation Strategies of Companies: A Comparative Institutional Approach of Some Cross-Country Differencies, in: Zapf, Wolfgang; Dierkes, Meinolf (Hg.), WZB-Jahrbuch 1994: Institutionenvergleich und Institutionendynamik, Berlin, 271-289.

Streeck, Wolfgang (1984): Industrial Relations in West Germany. A Case Study of the Car Industry, London.

Thelen, Kathleen A. (1992): Union of Parts. Labor Politics in Postwar Germany, Ithaca, London.

Thelen, Kathleen A.; Turner, Lowell (1997): German Codetermination in a Comparative Perspective, Gütersloh.

Wellhöner, Volker (1996): »Wirtschaftswunder, Weltmarkt, Westdeutscher Fordismus«. Der Fall Volkswagen, Münster.

Witte, Eberhard (1973): Organisation für Innovationsentscheidungen. Das PromotorenModell, Göttingen.

Womack, James P.; Jones, Daniel T.; Ross, Daniel (1990): The Machine that Changed the World, New York. 